\title{
Fangchinoline suppresses the proliferation, invasion and tumorigenesis of human osteosarcoma cells through the inhibition of PI3K and downstream signaling pathways
}

\author{
XIUCHENG LI ${ }^{1 *}$, ZHIFAN YANG ${ }^{1,2^{*}}$, WEIQI HAN ${ }^{1}$, XUANYUAN LU ${ }^{1}$, \\ SONGTAO JIN ${ }^{1}$, WANLEI YANG ${ }^{1}$, JIANLEI LI ${ }^{1}$, WEI HE ${ }^{1}$ and YU QIAN ${ }^{1}$ \\ ${ }^{1}$ Department of Orthopaedics, Shaoxing People's Hospital, Shaoxing Hospital of Zhejiang University, \\ Shaoxing, Zhejiang 312000; ${ }^{2}$ The Second Affiliated Hospital and Yuying Children's Hospital \\ of Wenzhou Medical University, Wenzhou, Zhejiang 325027, P.R. China
}

Received June 12, 2016; Accepted May 23, 2017

DOI: $10.3892 / \mathrm{ijmm} .2017 .3013$

\begin{abstract}
Osteosarcoma is the most common malignant bone tumor. Most patients diagnosed with osteosarcoma are less than 20 years of age. Osteosarcoma cells proliferate rapidly and invade other tissues. At present, neoadjuvant chemotherapy is the primary pharmacodynamic strategy to prevent the progression of osteosarcoma. However, adverse effects of this strategy limit its long-term application. Previous research has shown that fangchinoline exerts antitumor effects on several types of tumor cells; however, its effect on osteosarcoma cells remains unknown. The present study evaluated the effects of fangchinoline on the proliferation, apoptosis, migration and invasion of osteosarcoma cells in vitro and on their tumorigenesis in vivo and determined the possible underlying mechanism of action. Fangchinoline-treated MG63 and U20S cells showed significantly decreased proliferation and significantly increased apoptosis. Fangchinoline markedly suppressed the migration and invasion of the MG63 cells. Fangchinoline-treated MG63 cells showed significantly decreased expression of phosphoinositide 3-kinase (PI3K) and $\mathrm{Akt}^{\mathrm{t}}{ }^{\text {-Thr308 }}$. Moreover, fangchinoline-treated MG63 cells showed downregulated expression of cyclin D1 and matrix metalloproteinase 2 and 9, which act downstream of PI3K, and upregulated expression of caspase- 3 and caspase-8. Furthermore, fangchinoline suppressed the growth of subcutaneous osteosarcoma tumors in Balb/c mice subcutaneously injected with osteosarcoma cells. These findings suggest that fangchinoline inhibits the progression of osteosarcoma by suppressing the proliferation, migration and invasion and by
\end{abstract}

Correspondence to: Professor Yu Qian, Department of Orthopaedics, Shaoxing People's Hospital, Shaoxing Hospital of Zhejiang University, 568 Zhongxing North Road, Shaoxing, Zhejiang 312000, P.R. China E-mail: doctor120@hotmail.com

*Contributed equally

Key words: fangchinoline, osteosarcoma, proliferation, apoptosis, invasion, phosphoinositide 3-kinase accelerating the apoptosis of osteosarcoma cells. In addition, our results suggest that the mechanism underlying the antitumor effects of fangchinoline involve the inhibition of PI3K and its downstream signaling pathways.

\section{Introduction}

Osteosarcoma is the most common primary malignant bone tumor (1). It is derived from primitive mesenchymal cells and commonly develops in the distal femur and proximal tibia (2). Osteosarcoma predominantly affects young people, in particular, adolescents aged below 20 years $(3,4)$. The prognosis of osteosarcoma patients is poor with an overall 5-year survival rate below $30 \%$ in cases showing relapse and metastasis (5).

At present, neoadjuvant chemotherapy is the predominant treatment for osteosarcoma. Neoadjuvant chemotherapy combines surgery with pre- or post-operative multi-agent chemotherapy. Most patients require intensive chemotherapy with a combination of drugs such as methotrexate, cisplatin, doxorubicin, ifosfamide and etoposide (6). This leads to chemotherapy toxicity in normal tissues and cellular chemoresistance, which prevent the long-term application of this treatment $(7,8)$. Introduction of several targeted therapeutic agents has markedly improved the results of preclinical and clinical trials on osteosarcoma (9). However, the high cost of these therapeutic agents limit their widespread application. Therefore, safe and effective therapeutic approaches are urgently needed for treating osteosarcoma.

Fangchinoline is a bisbenzylisoquinoline alkaloid with a complex structure (Fig. 1A) and is extracted from an alkaloid tetrandrine, found in a traditional Chinese medicinal plant belonging to the Menispermaceae family. Increasing evidence suggests that fangchinoline posesses various pharmacological activities $(10,11)$. Current research has focused on the antitumor effects of fangchinoline and has shown that it exerts antitumor effects on lung cancer $(12)$, breast cancer $(13,14)$, hepatocellular carcinoma (15), chronic myelogenous leukemia (16), glioblastoma (17), gastric cancer (18), and prostate cancer cells (19). However, the antitumor effects of fangchinoline on osteosarcoma cells are unclear. 
Therefore, the present study was designed to evaluate the effects of fangchinoline on osteosarcoma cell lines MG63 and U20S. The study evaluated the effects of fangchinoline on the proliferation, apoptosis, migration and invasion of osteosarcoma cells in vitro and on their tumorigenesis in vivo and determined the possible underlying mechanism of action.

\section{Materials and methods}

Cell culture. Human osteosarcoma cell lines MG63 and U20S were purchased from the Cell Bank of the Chinese Academy of Sciences (Shanghai, China). The cells were cultured in Dulbecco's modified Eagle's medium (DMEM; Gibco BRL, Gaithersburg, MD, USA) supplemented with $10 \%$ fetal bovine serum (FBS) and $1 \%$ penicillin-streptomycin in an incubator with a humidified atmosphere of $5 \% \mathrm{CO}_{2}$. Cell medium was replaced with fresh medium containing dimethyl sulfoxide (DMSO) or 10, 20 or $30 \mu \mathrm{M}$ fangchinoline for the following experiments. Changes in the morphology of the cells were determined using an inverted phase-contrast microscope.

Cell proliferation assay. Cell viability was evaluated by performing 3-(4,5-dimethylthiazol-2-yl)-2,5-diphenyltetrazolium bromide (MTT) assay, as described previously (20). Briefly, MG63 or U20S cells were seeded in 96-well plates (cell density, $1 \times 10^{4}$ cells/well) containing $0.1 \mathrm{ml}$ medium supplemented with the indicated concentration of fangchinoline and were incubated for 24, 48 and $96 \mathrm{~h}$. At each time point, $0.01 \mathrm{ml} \mathrm{MTT}$ solution ( $5 \mathrm{mg} / \mathrm{ml}$ in PBS) was added to each well, and the cells were incubated for $4 \mathrm{~h}$ at $37^{\circ} \mathrm{C}$. The medium was replaced with DMSO for $10 \mathrm{~min}$ to solubilize crystals, and optical densities (ODs) were measured at $570 \mathrm{~nm}$.

Hoechst 33258 staining. MG63 cells were treated with DMSO or 10,20 or $30 \mu \mathrm{M}$ fangchinoline for $24 \mathrm{~h}$. Next, the cells were fixed with $4 \%$ polyoxymethylene, washed twice with PBS, and then incubated with $10 \mu \mathrm{g} / \mathrm{ml}$ Hoechst 33258 solution in the dark for $5 \mathrm{~min}$ at room temperature. Finally, the cells were washed three times with PBS and were observed under a fluorescence microscope.

Flow cytometry. Cell apoptosis was assessed using Annexin V-fluorescein isothiocyanate or Annexin V-propidium iodide (Annexin V-FITC/Annexin V-PI) apoptosis detection kit (Thermo Fisher Scientific, Waltham, MA, USA). MG63 cells were harvested, stained with Annexin V-FITC or Annexin V-PI, and analyzed using a FACScan flow cytometer (Becton-Dickinson, Franklin Lakes, NJ, USA). Cells showing Annexin V(+)/PI(-) staining were considered as early apoptotic cells and those showing Annexin $\mathrm{V}(+) / \mathrm{PI}(+)$ staining were considered as late apoptotic cells.

Scratch wound healing assay. MG63 cells were cultured in 6-well plates until confluency and were incubated in serum-free DMEM overnight before wounding. Scratches were made using a pipette tip, and the cells were treated with a medium containing DMSO or 10,20 or $30 \mu \mathrm{M}$ fangchinoline at $37^{\circ} \mathrm{C}$ for 24 and $48 \mathrm{~h}$. Images of the wounded area were obtained and quantified using an optical microscope.
Transwell assay. Transwell assay was performed to evaluate cell invasion. The upper part of each Transwell chamber was coated with $1 \times 10^{5}$ cells cultured in $0.1 \mathrm{ml}$ serum-free DMEM supplemented with DMSO or 10, 20 or $30 \mu \mathrm{M}$ fangchinoline, and the lower part of the chamber was filled with $0.6 \mathrm{ml}$ DMEM containing 10\% FBS. After incubation for $24 \mathrm{~h}$ at $37^{\circ} \mathrm{C}$, the cells in the upper part of chamber were removed. Cells invading the lower part of the chamber were fixed, stained, and counted using a high-power microscope.

Western blot analysis. Total cellular proteins were extracted from $1 \times 10^{6}$ cultured cells using $100 \mu \mathrm{l}$ RIPA lysis buffer. Next, $60 \mu \mathrm{g}$ of the protein samples were resolved by performing SDS-PAGE and were transferred onto nitrocellulose membranes through electroblotting. The membranes were blocked using $5 \%$ non-fat dry milk for $1 \mathrm{~h}$ and were probed by incubating overnight with the primary antibodies at $4^{\circ} \mathrm{C}$. Next, the membranes were washed and incubated with HRP-conjugated secondary antibodies (Sigma-Aldrich, St. Louis, MO, USA) for $1 \mathrm{~h}$. Immunoreactivity was detected using Western Lighting Ultra (ECL; Pierce Technology, Rockford, IL, USA). The following primary antibodies were used: i) rabbit polyclonal antibody against caspase-9 (ab32068) and monoclonal antibody against caspase-3 (ab32351) (Abcam, Cambridge, UK) at a dilution of 1:5,000; ii) rabbit polyclonal antibody against GAPDH (sc-25778) and monoclonal antibodies against phosphoinositide 3-kinase (PI3K; sc-7175), Akt (sc-135829), and Akt ${ }^{\mathrm{p}-\mathrm{Thr} 308}$ (sc-135650) (Santa Cruz Biotechnology, Inc., Santa Cruz, CA, USA) at a dilution of 1:1,000; and iii) rabbit monoclonal antibodies against cyclin D1 (60186-1-1g), MMP-2 (10373-2-AP), and MMP-9 (10375-2-AP) (ProteinTech Group, Inc., Rosemont, IL, USA) at a dilution of 1:1,000.

Animal model of osteosarcoma. Six-week-old female Balb/c-nu-nu mice were purchased from Guangxi Medical University (Guangxi, China). All animal experimental procedures were approved by the Ethics Committee (2016/025) of the Shaoxing People's Hospital (Zhejiang, China) and were performed as described previously (12). The mice were randomly divided into fangchinoline treatment and control groups, with six mice in each group. All the mice were maintained under sterile conditions at $20-25^{\circ} \mathrm{C}$ room temperature, $50-60 \%$ relative humidity, and a $12 \mathrm{~h}$ light/dark cycle and were fed a sterilized diet. After habituation for 7 days, the mice were anesthetized by intraperitoneally injecting $12 \mathrm{mg} / \mathrm{kg}$ chloral hydrate. Next, $0.2 \mathrm{ml}$ of the U20S cell suspension (cell density, $1 \times 10^{7}$ cells $/ \mathrm{ml}$ ) was subcutaneously injected into the left armpit of each mouse. Tumors became palpable 1 week after xenografting. Next, $0.1 \mathrm{ml}$ fangchinoline solution $(0.5 \mathrm{mg} / \mathrm{ml})$ or $0.1 \mathrm{ml}$ DMSO solution was locally injected into the tumors of each mouse three times per week. The mice were sacrificed 4 weeks after xenografting, and their tumors were harvested and weighed. The length and width of the tumors were measured to calculate tumor volume (tumor volume $=$ length $\mathrm{x}$ width ${ }^{2} / 2$ ).

Histological staining. The kidneys and livers of the mice were harvested, fixed, and embedded in paraffin. Histological sections were prepared for hematoxylin and eosin (H\&E) staining, and images were obtained using a light microscope. 
A

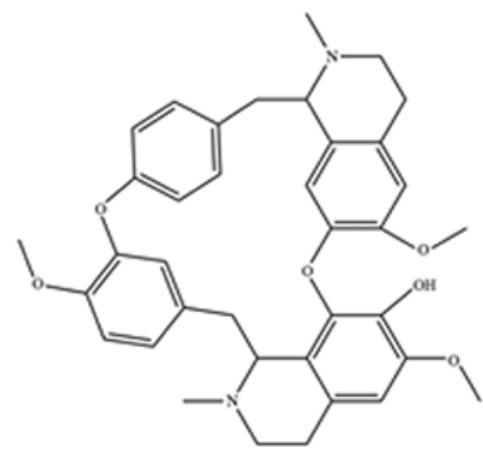

Fangchinoline

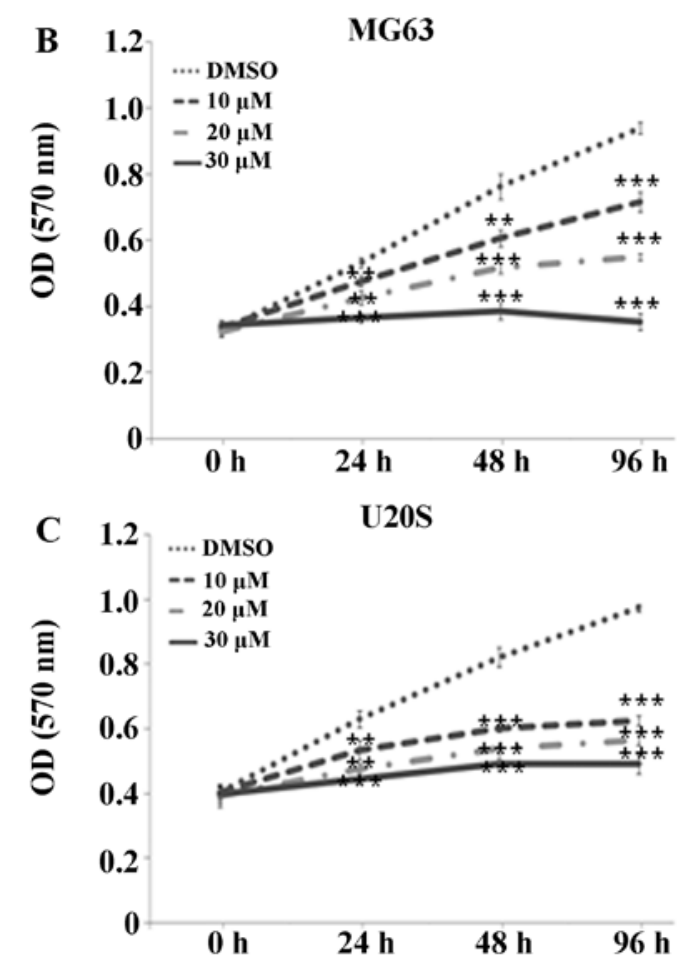

Figure 1. Fangchinoline suppresses the proliferation of osteosarcoma cells. (A) Molecular structure of fangchinoline. MG63 (B) and U20S cells (C) were cultured with the indicated concentrations of fangchinoline for the indicated times in 96 -well plates. Cell viability was determined by performing the MTT assay, and results are expressed as mean \pm SD of three experiments performed in triplicate. ${ }^{* *} \mathrm{P}<0.01$ and ${ }^{* * * *} \mathrm{P}<0.001$.

Statistical analysis. Data for all groups are presented as mean \pm SD. Differences between the groups were evaluated using one-way analysis of variance (ANOVA) with LSD test, and $\mathrm{P}<0.05$ was considered statistically significant. Statistical analysis was performed using SPSS software version 19.0.

\section{Results}

Fangchinoline decreases the viability of osteosarcoma cells. Fangchinoline markedly suppressed the proliferation of human osteosarcoma cell lines MG63 and U20S in a dose-dependent manner. The MTT assay showed that the ODs of the MG63 cells treated with 10,20 and $30 \mu \mathrm{M}$ fangchinoline significantly decreased to $0.72 \pm 0.03(\mathrm{P}<0.001), 0.55 \pm 0.01(\mathrm{P}<0.001)$, and $0.35 \pm 0.03(\mathrm{P}<0.001)$, respectively, after $96 \mathrm{~h}$ compared with the OD of the control cells $(0.94 \pm 0.02$; Fig. 1B). Similar trends were observed at the 24 and $48 \mathrm{~h}$ time points. The experiment was repeated using U20S cells, which responded similarly to the inhibitory effects of fangchinoline (Fig. 1C).

Fangchinoline increases the apoptosis of osteosarcoma cells. Fangchinoline treatment significantly accelerated the apoptosis of MG63 and U20S cells in a dose-dependent manner. Fangchinoline-untreated MG63 and U20S cells were rhombus-like and angular, as determined by performing inverted phase-contrast microscopy, and were attached to the culture plates along with few dead cells that were round and suspended (Fig. 2A). Treatment with 10, 20 and $30 \mu \mathrm{M}$ fangchinoline markedly increased the number of apoptotic cells to $177 \pm 19.1(\mathrm{P}<0.001), 322 \pm 21.5(\mathrm{P}<0.001)$, and $418 \pm 8.9$ $(\mathrm{P}<0.001)$, respectively, compared with that in the control cells (8.7 \pm 5.7 ; Fig. 2B). Similar trends were observed for U20S cells (Fig. 2C and D). Hoechst 33258 staining showed that fangchinoline treatment of MG63 cells induced the formation of apoptotic nuclei and condensed their chromatin, which appeared bright blue (Fig. 2E). Results of the flow cytometry showed that the percentages of late apoptotic cells increased to $10.35 \pm 0.55(\mathrm{P}=0.001), 12.77 \pm 0.69(\mathrm{P}<0.001)$ and $30.56 \pm 0.80 \%$ $(\mathrm{P}<0.001)$ in the 10,20 and $30 \mu \mathrm{M}$ fangchinoline-treated MG63 cells, respectively, compared with $7.23 \% \pm 0.15 \%$ in the control cells (Fig. 2F). A similar trend was observed for the percentages of early apoptotic MG63 cells.

Fangchinoline suppresses the migration and invasion of MG63 cells. Fangchinoline significantly inhibited the migration and invasion of MG63 cells in a dose-dependent manner. Distances traveled by 10,20 and $30 \mu \mathrm{M}$ fangchinoline-treated MG63 cells decreased to $0.86 \pm 0.03$ ( $\mathrm{P}<0.001), 0.50 \pm 0.03(\mathrm{P}<0.001)$, and $0.43 \pm 0.03 \mathrm{~mm}(\mathrm{P}<0.001)$, respectively, compared with the distance travelled by the control cells $(1.18 \pm 0.02 \mathrm{~mm})$ at the $48 \mathrm{~h}$ time point (Fig. 3A and B). Similar trends were observed at the $24 \mathrm{~h}$ time point. The Transwell assay showed that the number of 10,20 and $30 \mu \mathrm{M}$ fangchinoline-treated MG63 cells traversing through the polycarbonate membrane decreased to $79 \pm 4.6(\mathrm{P}<0.001), 44 \pm 7.0(\mathrm{P}<0.001)$ and $30 \pm 3.5$ $(\mathrm{P}<0.001)$, respectively, compared with the number of control cells (115 \pm 4.5 ; Fig. $3 \mathrm{C}$ and D).

Fangchinoline inhibits the PI3K/Akt signaling pathway. Fangchinoline treatment markedly decreased PI3K and Akt $\mathrm{p}^{\mathrm{p} \text {-Thr308 }}$ expression in a dose-dependent manner but did not alter the total Akt level. Moreover, fangchinoline treatment markedly decreased the level of cell proliferation regulator cyclin D1 but obviously increased the expression of caspase- 9 and caspase- 3 . In contrast, fangchinoline treatment significantly decreased MMP-2 and MMP-9 expression in the MG63 cells (Fig. 4A-C).

Fangchinoline inhibits the tumorigenesis of MG63 cells in vivo. The average size $\left(178.23 \pm 23.28 \mathrm{~mm}^{3}, \mathrm{P}=0.043\right)$ and weight $(0.19 \pm 0.03 \mathrm{~g}, \mathrm{P}=0.034)$ of tumors in the fangchinoline-treated mice were significantly decreased compared with these parameters in the control mice $\left(307.31 \pm 43.29 \mathrm{~mm}^{3}\right.$ and $0.33 \pm 0.04 \mathrm{~g}$, respectively; Fig. $5 \mathrm{~A}-\mathrm{C}$ ). In addition, none of the mice died during the experimental period. Moreover, no histological impact was observed on the kidneys and livers of mice in both the fangchinoline and control groups (Fig. 5D). 
A

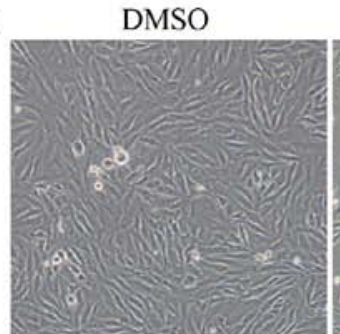

$20 \mu \mathrm{M}$

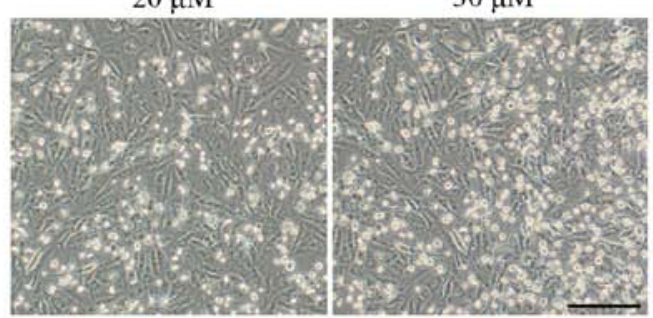

B

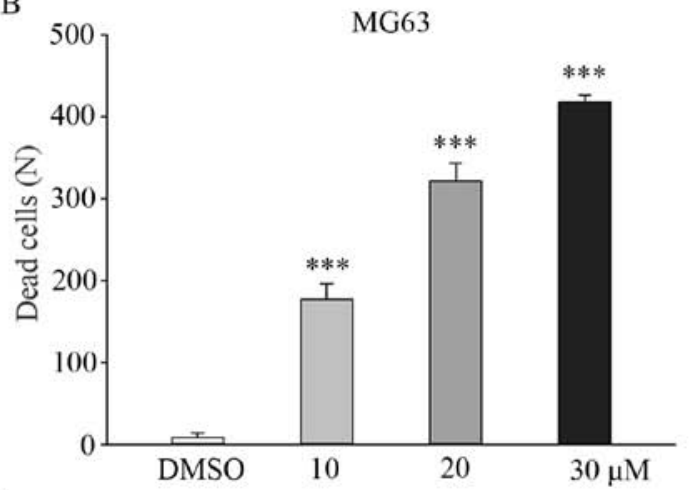

E

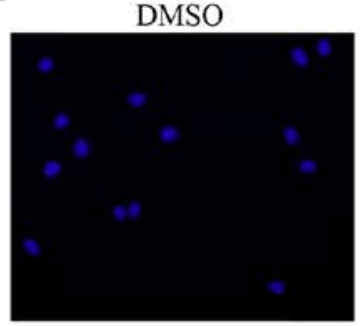

$\mathrm{F}$

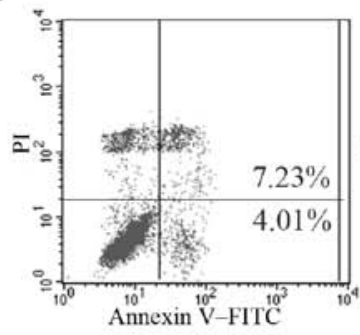

DMSO

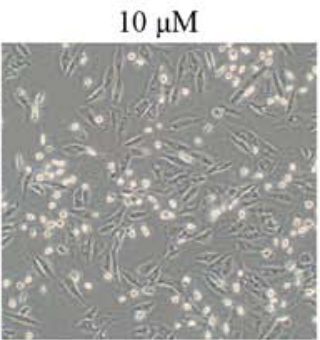

$30 \mu \mathrm{M}$
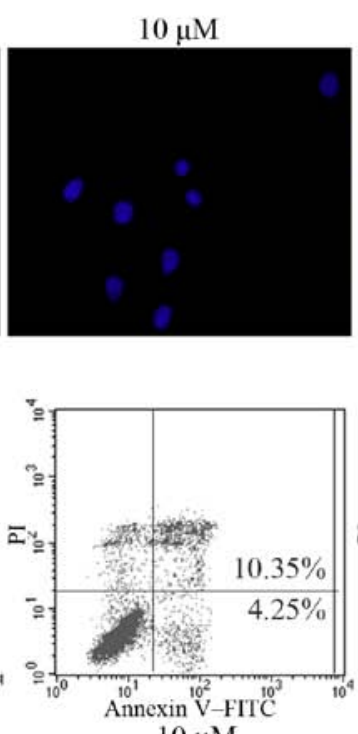

$10 \mu \mathrm{M}$

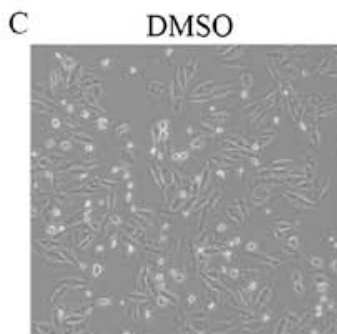

$20 \mu \mathrm{M}$

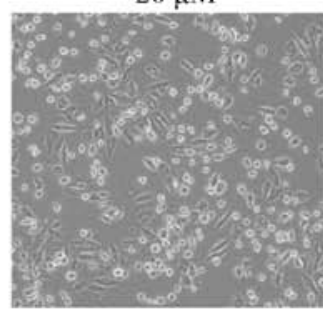

D

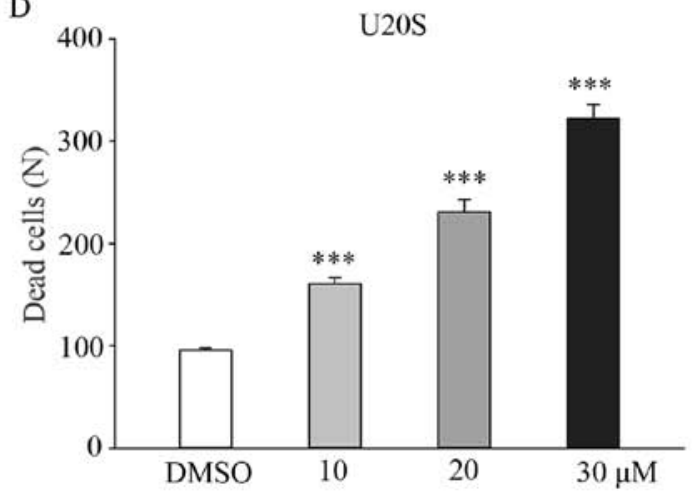

$20 \mu \mathrm{M}$
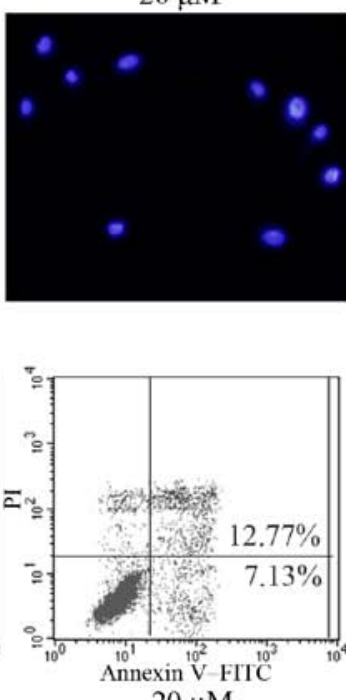

$20 \mu \mathrm{M}$
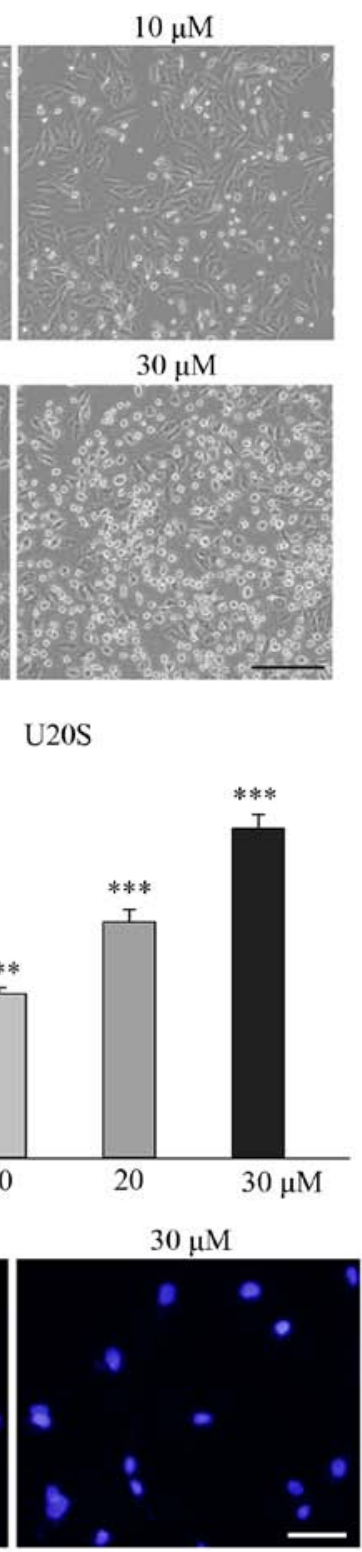

$30 \mu \mathrm{M}$

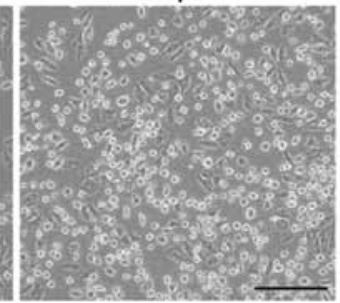

U20S

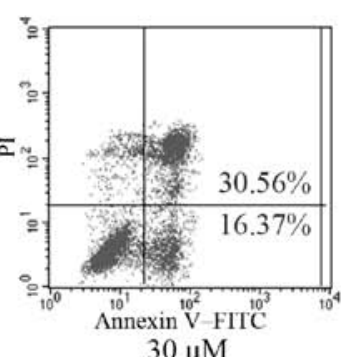

Figure 2. Fangchinoline increases the apoptosis of osteosarcoma cells. (A) Changes in the morphology of MG63 cells treated with DMSO or 10,20 or $30 \mu \mathrm{M}$ fangchinoline for $48 \mathrm{~h}$ were determined using an inverted phase-contrast microscope; scale bar, $100 \mu \mathrm{m}$. (B) Dead MG63 cells were counted, and results are presented as mean $\pm \mathrm{SD}$. (C) Changes in the morphology of U20S cell treated with DMSO or 10, 20 or $30 \mu \mathrm{M}$ fangchinoline for $48 \mathrm{~h}$ were determined using an inverted phase-contrast microscope; scale bar, $100 \mu \mathrm{m}$. (D) Dead U20S cells were counted, and results are presented as mean \pm SD; ${ }^{* * *} \mathrm{P}<0.001$. (E) MG63 cells were preincubated with DMSO or 10, 20 or $30 \mu \mathrm{M}$ fangchinoline for $48 \mathrm{~h}$. Next, the cells were stained with Hoechst 33258 solution and were observed under a fluorescence microscope; scale bar, $50 \mu \mathrm{m}$. (F) Flow cytometric analysis of MG63 cells treated with $0,10,25$ or $30 \mu \mathrm{M}$ fangchinoline for $24 \mathrm{~h}$. Annexin V(+)/ $\mathrm{PI}(-)$ cells were considered as early apoptotic cells.

\section{Discussion}

Previous studies have reported that fangchinoline exerts antitumor effects on several tumor cell lines by inhibiting proliferation and by inducing apoptosis. However, its effects on osteosarcoma cells have not been elucidated to date. In the present study, we observed that fangchinoline suppressed the proliferation, migration and invasion of osteosarcoma 

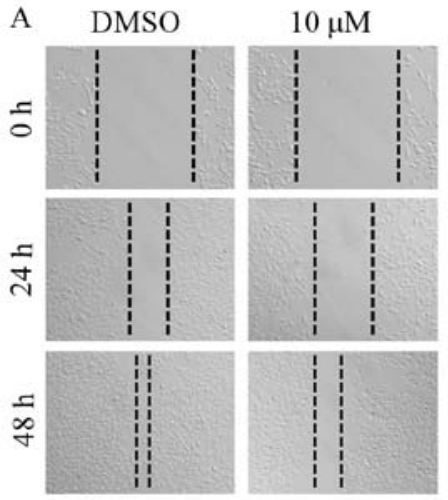

C
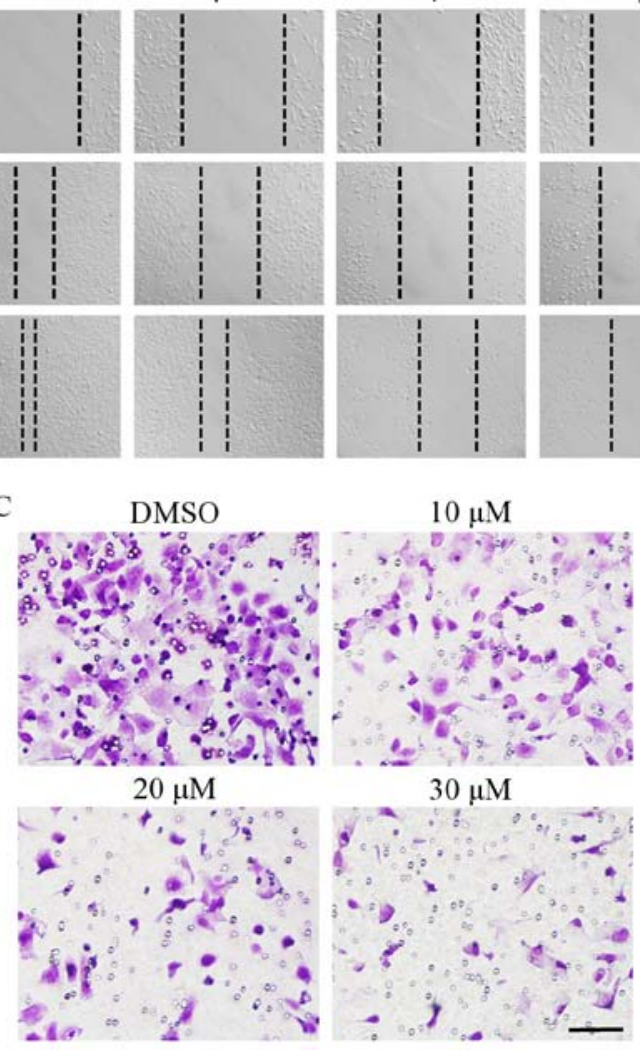

$30 \mu \mathrm{M}$
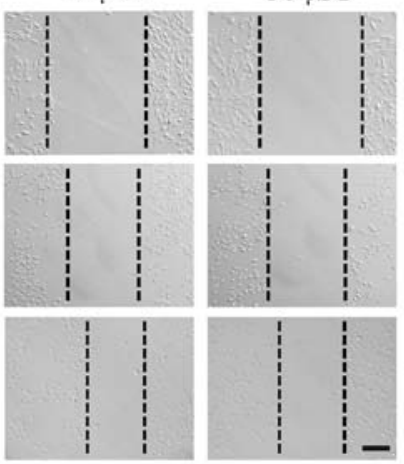

B
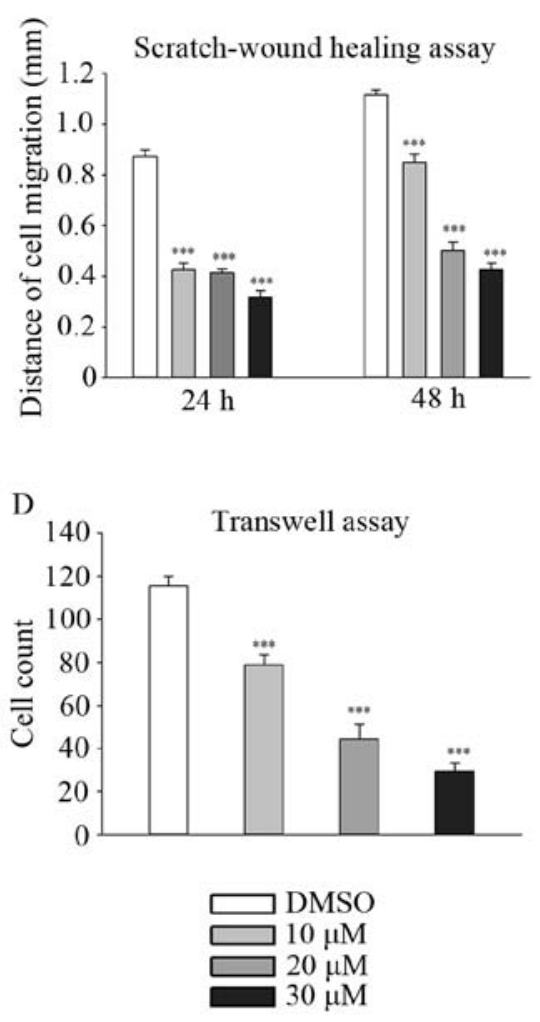

Figure 3. Fangchinoline inhibits the migration and invasion of osteosarcoma cells. (A) Representative microscopic images of the scratch wound healing assay showing the migration of MG63 cells treated with DMSO alone or with the indicated concentrations of fangchinoline for 24 and $48 \mathrm{~h}$; scale bar, $100 \mu \mathrm{m}$. (B) Measurement of cell migration distance. (C) Invasion of MG63 cells treated with DMSO or 10, 20 or $30 \mu \mathrm{M}$ fangchinoline for $48 \mathrm{~h}$ was determined by performing the Transwell assay; scale bar, $100 \mu \mathrm{m}$. (D) Cells that passed through the polycarbonate membrane were counted, and results are presented as mean $\pm \mathrm{SD} .{ }^{* * *} \mathrm{P}<0.001$.

\section{A}
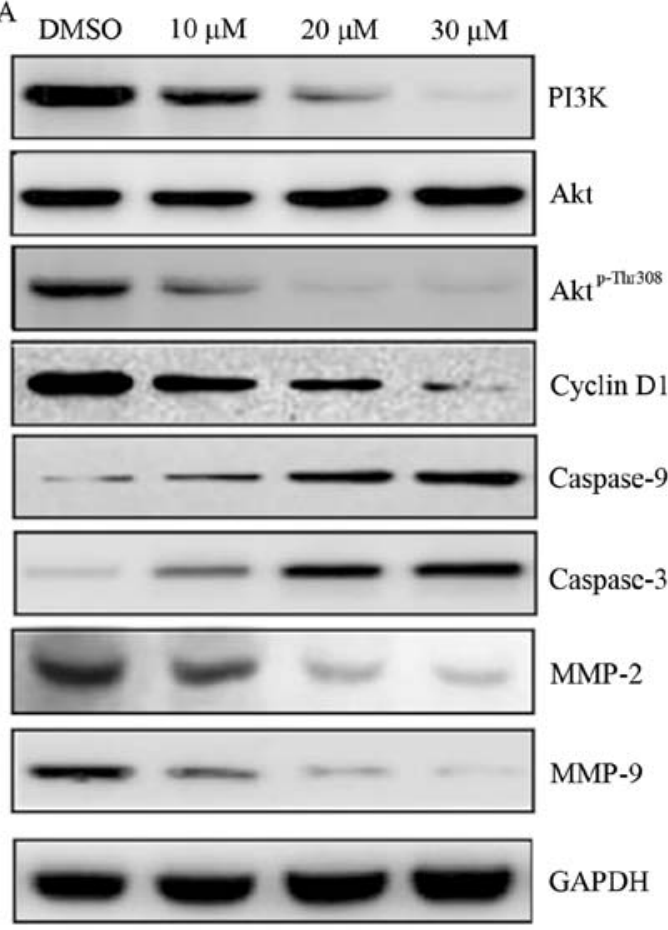
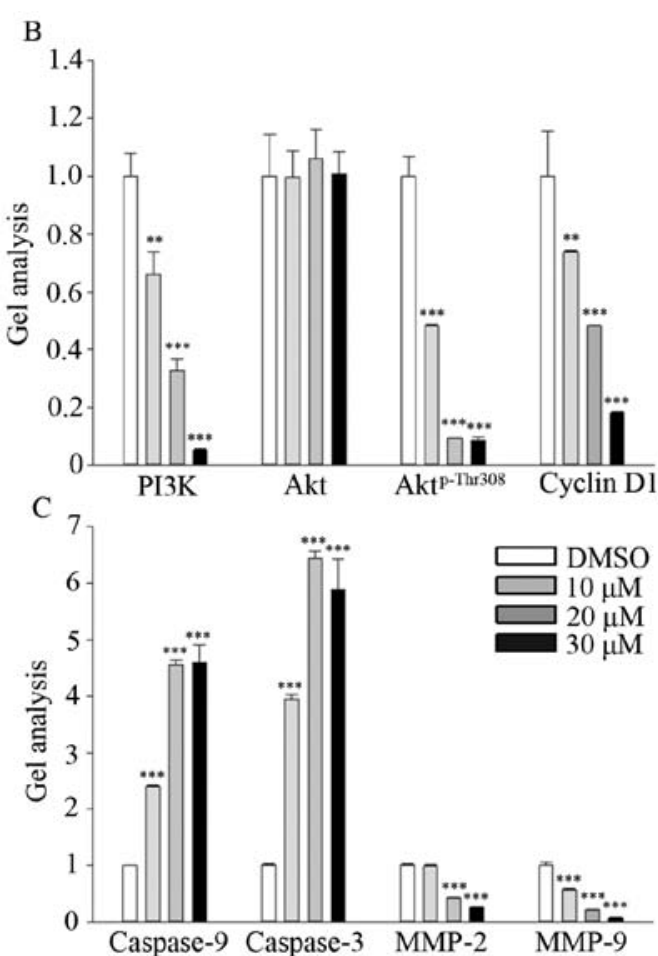

Figure 4. Fangchinoline inhibits the function of osteosarcoma cells by inhibiting the PI3K/Akt signaling pathway. (A) MG63 cells were treated with DMSO alone or with the indicated concentrations of fangchinoline for $48 \mathrm{~h}$. Expression of PI3K, Akt, Akt ${ }^{\text {-Thr308 }}$, cyclin D1, caspase-9, caspase-2, MMP-2, MMP-9, and GAPDH was detected by western blot analysis. (B and C) Results of quantitative western blot analysis were normalized using the protein expression profile of DMSO-treated cells. Data are presented as mean \pm SD. ${ }^{* *} \mathrm{P}<0.01$ and ${ }^{* * *} \mathrm{P}<0.001$. PI3K, phosphoinositide 3-kinase. 

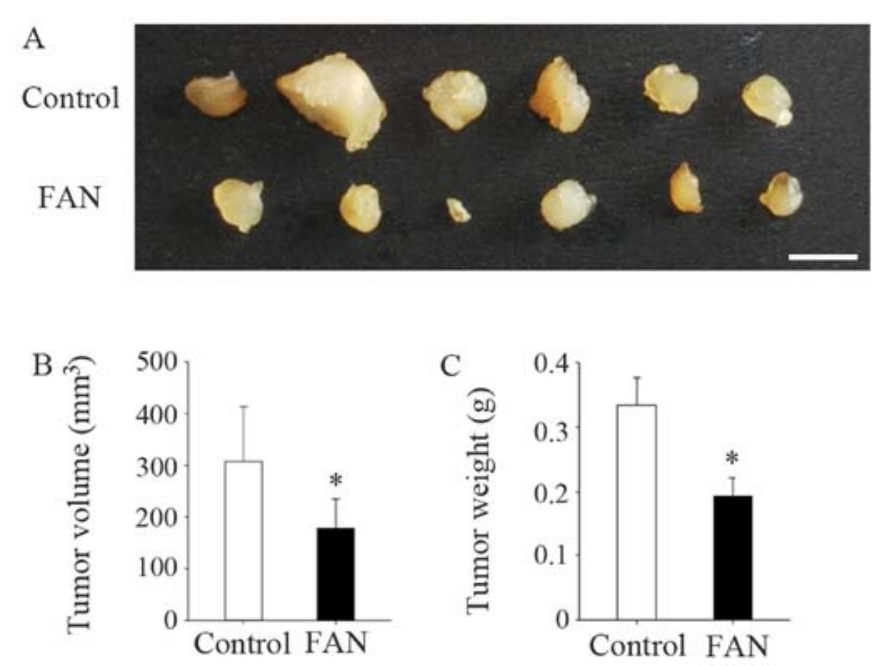

D

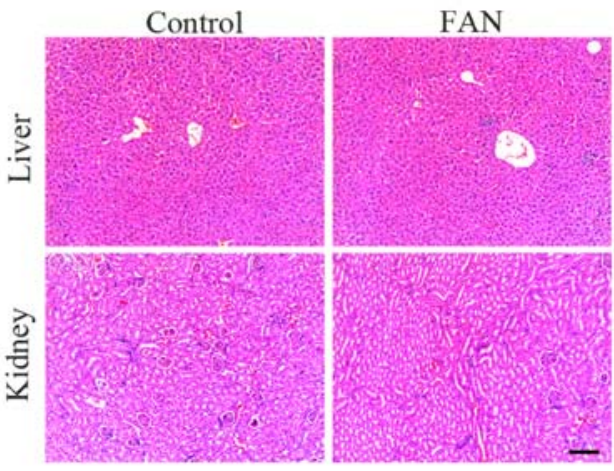

Figure 5. Fangchinoline inhibits the tumorigenesis of U20S cells in vivo. (A) Representative images of tumors excised from the fangchinoline-treated and control nude mice at 4 weeks after xenografting; scale bar, $1 \mathrm{~cm}$ (B) Tumor volumes and (C) tumor weights were measured after separation. Data are presented as mean \pm SD. ${ }^{*} \mathrm{P}<0.05$. (D) Representative images showing H\&E-stained livers (upper) and kidneys (bottom) of the control and fangchinoline-treated mice. Scale bar, $100 \mu \mathrm{m}$. FAN, fangchinoline.

cells lines MG63 and U20S in a dose-dependent manner. We speculate that these effects are associated with the inhibition of PI3K and its downstream signaling pathways. Moreover, we observed that fangchinoline inhibited the growth of subcutaneous osteosarcoma tumors in vivo. These results suggest that fangchinoline exerts antitumor effects against osteosarcoma cell lines.

Chemotherapeutic drugs such as paclitaxel exert an obvious killing effect on osteosarcoma cells (21). However, drug resistance and toxic side-effects after long-term application remain the main obstacles for their clinical use. Fangchinoline is a well-known traditional Chinese medicine that is chemically extracted from the alkaloid tetrandrine. It is an effective agent for tumor treatment and prevention, with no significant toxicity or side-effects (18). Research on the functions of fangchinoline will provide a potential new treatment for osteosarcoma, with important clinical significance.

The PI3K/Akt signaling pathway mediates various cellular functions, including cell proliferation, apoptosis and invasion. The PI3K/Akt pathway plays a central role in regulating the functions of osteosarcoma cells (22) and is an important target for treating different tumors (23-25). Fangchinoline was found to effectively suppress the proliferation of SGC7901, MKN45, and human gastric cancer cell lines that highly express PI3K

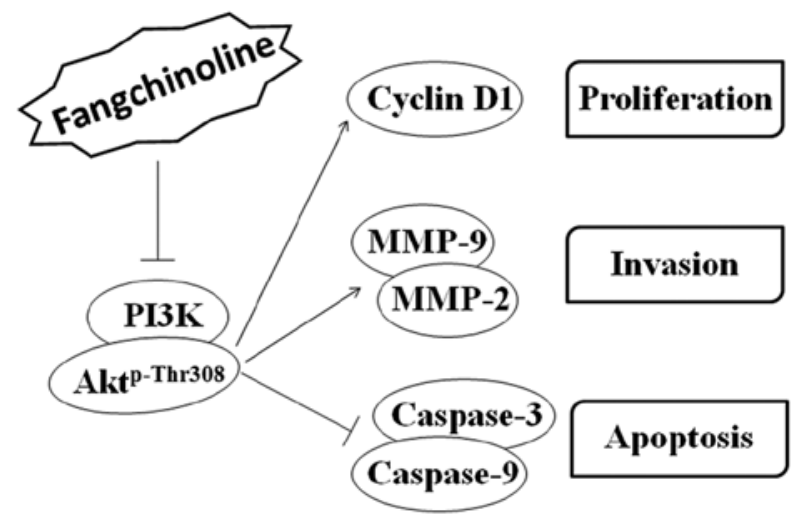

Figure 6. A schematic diagram showing that fangchinoline effectively suppresses proliferation and invasion and induces apoptosis by inhibiting PI3K and then suppressing PI3K-mediated signaling cascades. PI3K, phosphoinositide 3-kinase.

but to exert a negligible effect on human embryonic kidney 293 cells and normal cells that express low levels of PI3K (18), suggesting that fangchinoline is a novel inhibitor of PI3K and exerts antitumor effects by suppressing PI3K. Consistently, we found that fangchinoline treatment significantly decreased the expression of PI3K and $\mathrm{Akt}^{\mathrm{p}-\mathrm{Thr} 308}$ in a dose-independent manner. These findings may explain why traditional Chinese medicines show negligible toxicity against normal cells. Tumor cells grow rapidly because of their activated cell cycle progression. Cyclin D1, a downstream target of the PI3K/Akt signaling pathway, is a key regulator of the G1-S checkpoint $(26,27)$. Previous studies have shown that fangchinoline inhibits cell cycle progression in breast cancer cells by inhibiting the Akt/GSK-3 $\beta$ /cyclin D1 signaling pathway (13). Moreover, levels of cyclin D3 and cyclin E and cyclin-dependent kinase 2, 4 and 6 are decreased and levels of their endogenous inhibitors $\mathrm{p} 21^{\mathrm{WAF} 1}$ and $\mathrm{p} 27^{\mathrm{Kip} 1}$ are increased in fangchinoline-treated breast cancer cells (14). In the present study, cyclin D1 expression markedly decreased after fangchinoline treatment. These results suggest that fangchinoline prevents cell cycle progression in osteosarcoma cells by inhibiting the PI3K/Akt/cyclin D1 signaling pathway, thereby inhibiting the proliferation of these cells.

Enhancement of tumor cell apoptosis is another effective strategy for treating tumors. Fangchinoline was found to promote the early apoptosis of human glioblastoma cells by inhibiting Akt-mediated Bax and caspase-9 expression (17), suggesting that fangchinoline serves as a caspase agonist and can be used as an alternative treatment for tumors. Interestingly, another study showed that fangchinoline did not induce apoptotic cell death but induced autophagic cell death in hepatoma cell lines HepG2 and PLC/PRF-5 through the p53/sestrin2/AMPK signaling pathway (15). These results suggest that fangchinoline induces cell death through different mechanisms and exerts specific effects on different tumor cells. In the present study, we observed that fangchinoline significantly accelerated the apoptosis of MG63 cells and markedly decreased the expression of caspase- 8 and caspase-3. However, further studies are needed to elucidate whether fangchinoline induces autophagic cell death in osteosarcoma cells. Therefore, it is reasonable to speculate 
that fangchinoline enhances the apoptosis of osteosarcoma cells.

Besides accelerated proliferation, osteosarcoma cells show early metastasis. During metastasis, MMPs play a major role in degrading the extracellular matrix, thus allowing tumor cells to migrate and accelerate metastatic progression (28). A previous study showed that fangchinoline effectively inhibited the proliferation and invasion of gastric cancer cell lines BGC823 and SGC7901 by inhibiting PI3K-induced MMP-9 and MMP-2 expression (18). Similarly, we observed that fangchinoline significantly suppressed the migration and invasion of MG63 cells by downregulating MMP-9 and MMP-2 expression. This finding suggests that fangchinoline can be used as an antimetastatic agent. Moreover, fangchinoline suppressed the migratory and invasive potential of lung cancer cell line A549 by inhibiting the FAK/MMP-9/MMP-2 pathway (12). However, it is unclear whether fangchinoline targets PI3K in A549 cells. These findings suggest that fangchinoline inhibits MMPs by inhibiting different upstream signaling pathways. As fangchinoline downregulated the expression of MMPs and PI3K/Akt in the present study, we speculate that fangchinoline inhibits the migration and invasion of osteosarcoma cells by inhibiting the PI3K/Akt/MMP-9/MMP-2 signaling pathway.

An animal model of osteosarcoma was used to evaluate the antitumor effect of fangchinoline in vivo. Fangchinoline was previously found to inhibit the growth of lung tumors formed by A549 cells in a nude mouse model (12). Similarly, fangchinoline significantly suppressed the growth of subcutaneous osteosarcoma tumors in the present study. Moreover, fangchinoline treatment did not exert any toxic side-effects, as evidenced by the consistent histology of the kidneys and livers of mice in both the fangchinoline-treatment and control groups.

However, the present study has several limitations. First, a positive control group was required, such as cisplatin-treated osteosarcoma cells. Second, fangchinoline was injected locally into the tumors of the mouse models of osteosarcoma in the present study; however, it is unclear whether intravenous or oral administration of fangchinoline will exert the same effect. Third, the results of the H\&E staining did not precisely show the effect of fangchinoline on the physiological functions of the livers and kidneys of treated mice. Moreover, further clinical trials are needed to assess whether systemic treatment with moderate doses of fangchinoline affects bone metabolism.

In conclusion, the present study showed that fangchinoline suppressed the proliferation, migration, and invasion and enhanced the apoptosis of osteosarcoma cells. In addition, fangchinoline inhibited the growth of osteosarcoma tumors in vivo by possibly inhibiting $\mathrm{PI} 3 \mathrm{~K}$ and $\mathrm{Akt}$ and their downstream signaling pathways (Fig. 6). Thus, fangchinoline may serve as an auxiliary therapeutic agent for treating osteosarcoma.

\section{Acknowledgements}

The present study was supported by the Natural Science Foundation of Zhejiang Province (nos. LQ16H160013 and LY15H060005) and the National Natural Science Foundation of China (no. 81572126).

\section{References}

1. Picci P: Osteosarcoma (osteogenic sarcoma). Orphanet J Rare Dis 2: 6, 2007.

2. Jones KB: Osteosarcomagenesis: Modeling cancer initiation in the mouse. Sarcoma 2011: 694136, 2011.

3. Stiller CA, Craft AW and Corazziari I: Survival of children with bone sarcoma in Europe since 1978: Results from the EUROCARE study. Eur J Cancer 37: 760-766, 2001.

4. Mirabello L, Troisi RJ and Savage SA: Osteosarcoma incidence and survival rates from 1973 to 2004: Data from the Surveillance, Epidemiology, and End Results Program. Cancer 115: 1531-1543, 2009.

5. Wu PK, Chen WM, Chen CF, Lee OK, Haung CK and Chen TH: Primary osteogenic sarcoma with pulmonary metastasis: Clinical results and prognostic factors in 91 patients. Jpn J Clin Oncol 39: 514-522, 2009.

6. Group TEES; ESMO/European Sarcoma Network Working Group: Bone sarcomas: ESMO Clinical Practice Guidelines for diagnosis, treatment and follow-up. Ann Oncol 25 (Suppl 3): iii113-iii123, 2014

7. Yang R, Sowers R, Mazza B, Healey JH, Huvos A, Grier H, Bernstein M, Beardsley GP, Krailo MD, Devidas M, et al: Sequence alterations in the reduced folate carrier are observed in osteosarcoma tumor samples. Clin Cancer Res 9: 837-844, 2003.

8. Guo W, Healey JH, Meyers PA, Ladanyi M, Huvos AG, Bertino JR and Gorlick R: Mechanisms of methotrexate resistance in osteosarcoma. Clin Cancer Res 5: 621-627, 1999.

9. Shaikh AB, Li F, Li M, He B, He X, Chen G, Guo B, Li D, Jiang F, Dang L, et al: Present advances and future perspectives of molecular targeted therapy for osteosarcoma. Int J Mol Sci 17: $506,2016$.

10. Ma W, Nomura M, Takahashi-Nishioka T and Kobayashi S: Combined effects of fangchinoline from Stephania tetrandra Radix and formononetin and calycosin from Astragalus membranaceus Radix on hyperglycemia and hypoinsulinemia in streptozotocin-diabetic mice. Biol Pharm Bull 30: 2079-2083, 2007.

11. Gülçin I, Elias R, Gepdiremen A, Chea A and Topal F: Antioxidant activity of bisbenzylisoquinoline alkaloids from Stephania rotunda: Cepharanthine and fangchinoline. J Enzyme Inhib Med Chem 25: 44-53, 2010.

12. Guo B, Su J, Zhang T, Wang K and Li X: Fangchinoline as a kinase inhibitor targets FAK and suppresses FAK-mediated signaling pathway in A549. J Drug Target 23: 266-274, 2015.

13. Wang CD, Yuan CF, Bu YQ, Wu XM, Wan JY, Zhang L, Hu $\mathrm{N}$, Liu XJ, Zu Y, Liu GL, et al: Fangchinoline inhibits cell proliferation via Akt/GSK-3beta/cyclin D1 signaling and induces apoptosis in MDA-MB-231 breast cancer cells. Asian Pac J Cancer Prev 15: 769-773, 2014

14. Xing Z, Zhang Y, Zhang X, Yang Y, Ma Y and Pang D: Fangchinoline induces G1 arrest in breast cancer cells through cell-cycle regulation. Phytother Res 27: 1790-1794, 2013.

15. Wang N, Pan W, Zhu M, Zhang M, Hao X, Liang G and Feng Y: Fangchinoline induces autophagic cell death via p53/sestrin2/AMPK signalling in human hepatocellular carcinoma cells. Br J Pharmacol 164: 731-742, 2011.

16. Wang Y, Chen J, Wang L, Huang Y, Leng Y and Wang G: Fangchinoline induces G0/G1 arrest by modulating the expression of CDKN1A and CCND2 in K562 human chronic myelogenous leukemia cells. Exp Ther Med 5: 1105-1112, 2013.

17. Guo B, Xie P, Su J, Zhang T, Li X and Liang G: Fangchinoline suppresses the growth and invasion of human glioblastoma cells by inhibiting the kinase activity of Akt and Akt-mediated signaling cascades. Tumour Biol 37: 2709-2719, 2016.

18. Tian F, Ding D and Li D: Fangchinoline targets PI3K and suppresses PI3K/AKT signaling pathway in SGC7901 cells. Int J Oncol 46: 2355-2363, 2015.

19. Li D, Lu Y, Sun P, Feng LX, Liu M, Hu LH, Wu WY, Jiang BH, Yang M, Qu XB, et al: Inhibition on proteasome $\beta 1$ subunit might contribute to the anti-cancer effects of fangchinoline in human prostate cancer cells. PLoS One 10: e0141681, 2015.

20. Li X, Huang T, Jiang G, Gong W, Qian H and Zou C: Synergistic apoptotic effect of crocin and cisplatin on osteosarcoma cells via caspase induced apoptosis. Toxicol Lett 221: 197-204, 2013.

21. Tsai HC, Huang CY, Su HL and Tang CH: CTGF increases drug resistance to paclitaxel by upregulating survivin expression in human osteosarcoma cells. Biochim Biophys Acta 1843: 846-854, 2014. 
22. Zhang J, Yu XH, Yan YG, Wang C and Wang WJ: PI3K/Akt signaling in osteosarcoma. Clin Chim Acta 444: 182-192, 2015.

23. Li YJ, Dong BK, Fan M and Jiang WX: BTG2 inhibits the proliferation and metastasis of osteosarcoma cells by suppressing the PI3K/AKT pathway. Int J Clin Exp Pathol 8: 12410-12418, 2015

24. Du S and Yang L: ClC-3 chloride channel modulates the proliferation and migration of osteosarcoma cells via AKT/GSK3 $\beta$ signaling pathway. Int J Clin Exp Pathol 8: 1622-1630, 2015.

25. Ma X, Sun W, Shen J, Hua Y, Yin F, Sun M and Cai Z: Gelsolin promotes cell growth and invasion through the upregulation of p-AKT and p-P38 pathway in osteosarcoma. Tumour Biol 37: $7165-7174,2016$
26. Nakagami H, Kawamura K, Sugisaka K, Sekine $M$ and Shinmyo A: Phosphorylation of retinoblastoma-related protein by the cyclin D/cyclin-dependent kinase complex is activated at the G1/S-phase transition in tobacco. Plant Cell 14: 1847-1857, 2002.

27. Keenan SM, Lents NH and Baldassare JJ: Expression of cyclin E renders cyclin D-CDK4 dispensable for inactivation of the retinoblastoma tumor suppressor protein, activation of $\mathrm{E} 2 \mathrm{~F}$, and G1-S phase progression. J Biol Chem 279: 5387-5396, 2004.

28. Libra M, Scalisi A, Vella N, Clementi S, Sorio R, Stivala F, Spandidos DA and Mazzarino C: Uterine cervical carcinoma: Role of matrix metalloproteinases (Review). Int J Oncol 34: 897-903, 2009. 